\title{
A UNIQUENESS THEOREM FOR SOME NONLINEAR BOUNDARY VALUE PROBLEMS ${ }^{1}$
}

\section{A. KADISH}

In an earlier paper, it was shown, using a modified energy integral technique, that the Dirichlet and Neumann boundary value problems for linear differential equations of a certain class always have a unique solution [1]. In this paper, a theorem is proved which extends the linear uniqueness theorem to some nonlinear boundary value problems.

In the following the domains of the variable $x=\left(x_{1}, x_{2}, \cdots, x_{n}\right)$ and the parameter $z$ are taken to be $X$ and $D$ respectively. Their boundaries are written $\partial X$ and $\partial D$. A subdomain of $D$, either proper or improper, is denoted by $D^{*}$. The set $U_{F}(\lambda, \mu)$ is defined to be the collection of pairs of complex numbers $\left(\lambda_{\alpha}, \mu_{\alpha}\right)$ which are such that

(i) $F\left(x, z, \lambda_{\alpha}, \mu_{\alpha}\right)$ is analytic in $z$ on $D \times X$ and

(ii) there exist two real valued functions $p_{\lambda}(x, z)$ and $p_{\mu}(x, z)$ defined on $X \times \partial D$ such that

$$
\begin{gathered}
\left|F\left(x, z, \lambda_{\alpha}, \mu_{\alpha}\right)-F(x, z, \lambda, \mu)\right| \\
<p_{\lambda}(x, z)\left|\lambda_{\alpha}-\lambda\right|+p_{\mu}(x, z)\left|\mu_{\alpha}-\mu\right| .
\end{gathered}
$$

In the following, the function $v\left(x, z_{0}\right)\left(z_{0} \in D^{*}\right)$ will be said to be $F^{(u)}$-admissible if $\left(v, \nabla_{x} v\right) \in U_{F}(u, \nabla u)$ and both $v$ and $\nabla_{x} v$ are square integrable $\left(\nabla_{x}=\left(\partial / \partial x_{1}, \partial / \partial x_{2}, \cdots, \partial / \partial x_{n}\right)\right)$. In the complex number plane, half planes whose boundaries contain the origin shall be denoted by $H$. The outward drawn unit normal to such a half plane is $v_{H}$.

The operator

$$
L=-\nabla_{x} \cdot a(x, z) \nabla_{x}+b(x, z)
$$

with $a(x, z)$ and $b(x, z)$ analytic in $z$ on $D \times X$ is of class $H_{0}$ if and only if

(i) for each $z \in \partial D$ and every pair of real numbers $(\xi, \eta) \neq(0,0)$, there exists an $H$ such that the mapping $h_{a b}(\xi, \eta)$ of $X$

$$
h_{a b}(\xi, \eta)=a(x, z) \xi^{2}+b(x, z) \eta^{2}
$$

is contained in the interior of $H$, and

Received by the editors September 14, 1967. 7543.

${ }^{1}$ This work was supported in part by National Science Foundation Contract GP 
(ii) the winding number (with respect to the origin of the complex plane) of $v_{H}$, defined as a continuous function of $z$ on $\partial D$, is zero.

Consider the equation

$$
L[u]=F\left(x, z, u, \nabla_{x} u\right) .
$$

Problem $\mathrm{N}$ will be to solve equation (4) on $D^{*} \times X$ together with a Neumann condition on $D^{*} \times \partial X$. Problem $\mathrm{D}$ will be to solve equation (4) on $D^{*} \times X$ together with a Dirichlet condition on $D^{*} \times \partial X$.

Theorem. Assume that $L \in H_{0}$. Assume that on $X \times \partial D$ there exists a real function $\theta(z)$, which is such that

$$
\begin{aligned}
& \frac{1}{2} p_{\mu}(x, z) \leqq \min _{x \in X} \operatorname{Re}\left\{a(x, z) e^{-i \theta(z)}\right\}, \\
& \frac{1}{2} p_{\mu}(x, z)+p_{\lambda}(x, z) \leqq \min _{x \in X} \operatorname{Re}\left\{b(x, z) e^{-i \theta(z)}\right\} .
\end{aligned}
$$

Then any $F^{(u)}$-admissible function $v(x, z)$ which solves either problem $\mathrm{N}$ or problem $\mathrm{D}$ is unique in $U_{F}(u, \nabla u)$ if $u$ is a solution.

Proof. Assume that an $F^{(u)}$-admissible function $u$ solves problem $\mathrm{N}$ (or D) and that there exists a second $F^{(u)}$-admissible solution $u_{0}$ for some value of $z_{0} \in D^{*}$. Then their difference $\psi\left(x, z_{0}\right)=u_{0}\left(x, z_{0}\right)$ $-u\left(x, z_{0}\right)$ solves the equation

$$
L(\psi)=F\left(x, z, u_{0}, \nabla_{x} u_{0}\right)-F\left(x, z, u, \nabla_{x} u\right)=F\left(x, z, u_{0}, u\right)
$$

when $z=z_{0}$, together with appropriate data equal to zero on $D^{*} \times \partial X$. Therefore, multiplying equation (6) by $\Psi\left(x, z_{0}\right)$, the complex conjugate of $\psi\left(x, z_{0}\right)$, and integrating by parts yields

$$
0=\int_{X} d x\left[\left\{h_{a b}\left(|\psi|,\left|\nabla_{x} \psi\right|\right)\right\}-\bar{\psi}\left(x, z, u_{0}, u\right)\right] .
$$

Define the function

$$
\begin{aligned}
S\left(z, z_{0}\right)= & \int_{X} d x\left\{a(x, z)\left|\nabla_{x} \psi\left(x, z_{0}\right)\right|^{2}+b(x, z)\left|\psi\left(x, z_{0}\right)\right|^{2}\right\} \\
& -\int_{X} d x\left\{\bar{\psi}\left(x, z_{0}\right) \mathcal{F}\left(x, z, u_{0}\left(x, z_{0}\right), u\left(x, z_{0}\right)\right\} .\right.
\end{aligned}
$$

Since $u_{0}$ and $u$ are admissible, $S\left(z, z_{0}\right)$ is analytic in $z$ on $D$. From equation (6) we conclude that $S\left(z_{0}, z_{0}\right)=0$. From equation (1), the absolute value of the second integral is less than

$$
\int_{x} d x\left[\left\{p_{\lambda}(x, z)+\frac{1}{2} p_{\mu}(x, z)\right\}\left|\psi\left(x, z_{0}\right)\right|^{2}+\left\{\frac{1}{2} p_{\mu}(x, z)\right\}\left|\nabla_{x} \psi\left(x, z_{0}\right)\right|^{2}\right]
$$


which is less than the absolute value of the first integral on $X \times \partial D$. By Rouché's theorem, the number of zeros of $S\left(z, z_{0}\right)$ and the number of zeros of the first integral in $D$ are the same. However, it has already been shown in an earlier paper that if $L \in H_{0}$, the first integral cannot vanish for any value of $z \in D$ when $\psi(x, z)$ is the difference of two admissible functions, unless $\psi\left(x, z_{0}\right)=0$ almost everywhere [1]. Thus, the solution is unique.

\section{REFERENCE}

1. A. Kadish, J. Math. Phys. 9 (1968), 1266.

UNIVERSITY OF WISCONSIN 